\section{HIF2A: mastering osteoarthritis}

\section{By Michael J. Haas, Senior Writer}

Most approved osteoarthritis therapies treat symptoms of the disease but not the underlying processes that lead to cartilage destruction and bone spur formation in joints. Now, separate research teams from Japan and South Korea have shown that the transcription factor endothelial PAS domain protein 1 is a key regulator of osteoarthritis pathogenesis. ${ }^{1,2}$

Both teams expect that endothelial PAS domain protein 1 (EPAS1; HIF2A) inhibitors could treat or prevent the disease, although the researchers differ on whether HIF2A itself or an upstream activator of the transcription factor would be a better drug target.

The normal growth of long bones and the development of OA both depend on endochondral ossification, a process that involves the growth, expansion and subsequent degradation of cartilage cells. However, the molecular factors that regulate and coordinate the processincluding what goes awry in $\mathrm{OA}$ - are not well understood.

HIF2A is a member of the family of hypoxia-inducible factors (HIFs) that are activated under hypoxic conditions. Although cartilage tissue is hypoxic, the role of HIF2A in hypoxic tumors and its dysfunction in polycythemia-the overproduction of red blood cells-has attracted greater attention from researchers than the potential role of the protein in endochondral ossification.

Both research teams set out to determine whether HIF2A plays a role in normal bone growth or OA pathogenesis. The Japanese team screened mouse and human cell lines for transcription factors that activated the secretion of collagen type X $\alpha-1$ (COL10A1) - an early step in endochondral ossification-and identified HIF2A as the most likely player.

Additional screening also showed that proinflammatory molecules such as NF- $\kappa B$, IL- $1 \beta$ and tumor necrosis factor- $\alpha$ (TNF- $\alpha$ ) were involved in HIF2A activation.

The South Korean team took a different approach. They screened joint cartilage cells from mice and human OA patients for genes that were upregulated by IL- $1 \beta$, TNF- $\alpha$ and other proinflammatory cytokines and subsequently identified the gene for HIF2A.

Both groups elucidated similar roles for HIF2A in OA. In mouse cartilage cells, Hif2a activated matrix metalloproteinase 3 (Mmp3),
Mmp9, Mmp13 and other molecules involved in cartilage degradation. Hif $2 a$ knockout mouse and rabbit models of knee OA exhibited less cartilage destruction than wild-type controls.

Also, HIF2A expression was higher in cartilage samples from OA patients than in samples from healthy individuals.

The South Korean team showed that transgenic mice overexpressing Hif $2 a$ had greater cartilage damage in knee joints than normal controls, and the Japanese team found a SNP on the gene for HIF2A that was associated with knee OA $(p=0.013)$.

Collectively, the two sets of data demonstrate that HIF2A in cartilage cells is a key regulator of both normal and osteoarthritic endochondral ossification (see Figure 1, "HIF2A: master switch in cartilage").

The Japanese team from The University of Tokyo first saw the Korean group's data at a symposium in October 2009 and was surprised by the agreement with their own findings, Tokyo team leader Hiroshi Kawaguchi told SciBX. "We are happy that our data are very clearly supported by the Korean paper" and demonstrate the potential utility of HIF2A as a drug target to treat OA, he said.

Kawaguchi is a professor of orthopedic surgery at the University of Tokyo.

South Korean team leader Jang-Soo Chun said his team's paper also showed that HIF2A in cartilage cells regulates the expression of inducible nitric oxide synthase 2 (NOS2; iNOS) and cyclooxygenase-2 (COX-2), which are involved in inflammatory and pain responses. As a result, he said, HIF2A inhibitors might help treat osteoarthritic inflammation and pain.

"HIF2A appears to act as a master transcription factor in osteoarthritis development," noted Chun. "Therefore, in terms of developing strategies to treat osteoarthritis, inhibition of HIF2A might be more effective than inhibiting individual downstream molecules" such as MMPs, iNOS or COX-2.

Chun is a professor at Gwangju Institute of Science and Technology's School of Life Sciences and director of the Cell Dynamics Research Center at Gwangju Institute. His team included researchers from Ajou University and Wonkwang University Medical Center.

Both groups reported their findings in Nature Medicine.

\section{Joint administration}

"Based on these two papers, companies should become aware of HIF2A as a potential osteoarthritis target and think about developing HIF2A inhibitors," said Thomas Pap, professor of experimental medicine and director of the Institute of Experimental Musculoskeletal Medicine at University Hospital Muenster.

He was especially interested in the Japanese team's paper because it described an idea to which Pap's research group subscribes: that OA involves the re-initiation of a process that normally occurs in embryos and children and ceases in adulthood. This idea implies that HIF2A 


\section{ANALYSIS}

\section{COVER STORY}

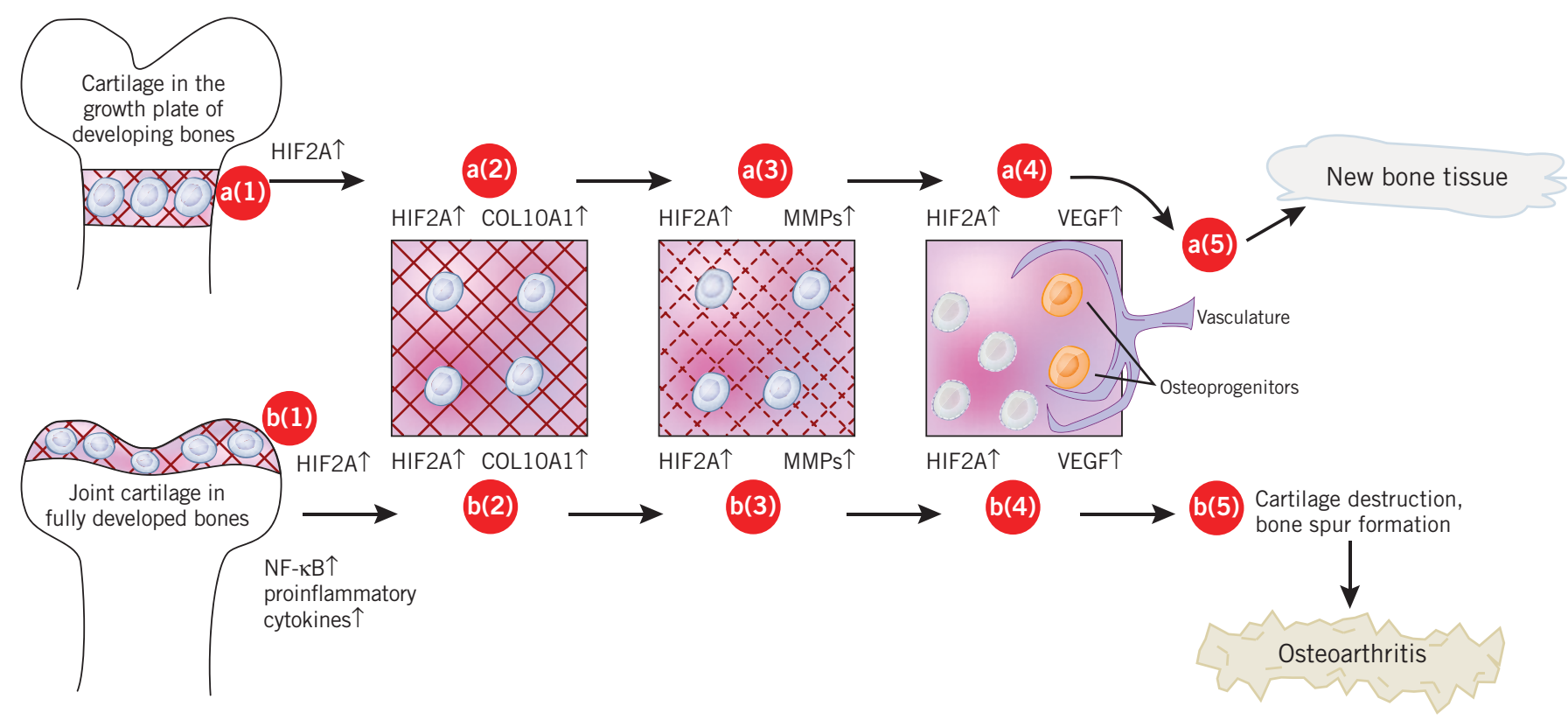

Figure 1. HIF2A: master switch in cartilage. Endothelial PAS domain protein 1 (EPAS1; HIF2A) expressed in cartilage cells regulates normal long bone growth and pathogenesis of osteoarthritis (OA).

In normal long bone growth, HIF2A is upregulated in the growing cartilage cells of bone growth plates [a(1)], inducing expression of collagen type $\mathrm{X} \alpha-1$ (COL10A1) [a(2)], which forms the cartilage matrix (solid red mesh), followed by matrix metalloproteinases (MMPs) that degrade the cartilage matrix (dotted red mesh) [a(3)]. Next, HIF2A induces VEGF, which promotes angiogenesis in the space left by degraded matrix and dying cartilage cells (dotted red mesh), causing infiltration of osteoprogenitor cells [a(4)] and subsequent formation of new bone tissue [a(5)].

In OA, proinflammatory cytokines such as NF-KB upregulate HIF2A in joint cartilage cells [b(1)], sequentially inducing them [b(2-4)] to cause pathological cartilage destruction and bone spur (osteophyte) formation [b(5)] instead of normal bone growth.

is not especially active in adult cartilage tissue, which in turn might explain why the protein's role in cartilage cells had not been previously explored, he said.

At the same time, Pap noted, "HIF2A in cartilage would be especially difficult to target with a systemic drug because cartilage lacks vasculature" and transcription factors are always difficult to target.

Moreover, he said, the two teams' findings showed that "HIF2A appears to coordinate and integrate signals from multiple biological pathways, so inhibiting factors up- or downstream from HIF2A might have a less complete effect on osteoarthritis than targeting HIF2A directly."

One solution to these challenges might be to administer a HIF2A inhibitor by intra-articular injection (direct injection into the joint), whereby joint cartilage cells would take up the inhibitor via diffusion from synovial fluid and surrounding joint tissue, he said.

To further validate HIF2A as a target to treat OA, Pap wanted to see inhibitors of HIF2A tested in wild-type mouse models of induced OA. Such experiments would exclude the possibility that Hif2a knockout had effects on the animals' development-such as altering cartilage structure or other factors-that made the cartilage less susceptible to degradation, he said.

Pap also noted that some disagreements between the Japanese and Korean teams' findings-such as whether HIF2A upregulates ADAM metallopeptidase with thrombospondin type 1 motif 4 (ADAMTS4) indicates that additional studies are needed to explore the specific mechanisms of HIF2A activation and its downstream effects.

"Understanding these mechanisms would be important to evaluating the potential side effects of HIF2A inhibition because of the range of metalloproteinases and other proteins that HIF2A appears to regulate," he said.

Chun suggested that the differing ADAMTS4 results were attributable to the different experimental systems used by each team. The Chun team's experiments in primary joint cartilage cell cultures showed that HIF2A activated ADAMTS4, whereas the Kawaguchi team's experiments in a mouse cartilage cell line showed that HIF2A did not activate ADAMTS4.

\section{Going upstream vs. direct}

The Tokyo team is now "searching for the extracellular signal that lies upstream of HIF2A" and either directly activates or suppresses it, because an extracellular protein might be easier to target than an intracellular transcription factor like HIF2A, Kawaguchi said.

Kawaguchi said his team has not patented the findings reported in Nature Medicine. He added that the team would be interested in licensing arrangements once it identifies the upstream activator or suppressor of HIF2A. 


\section{ANALYSIS}

In contrast, Chun said, the South Korean team's ongoing work includes screening for potential HIF2A inhibitors.

Chun agreed that intracellular transcription factors are challenging targets but added that the team is screening compounds that permeate the cell membrane. "Also, we hope that we can find compounds which interact with HIF2A to prevent it from being imported into the nucleus or that would bind HIF2A in the cytosol and inhibit its activity regardless of nuclear import," he said.

Nevertheless, Chun also agreed that blocking HIF2A expression was another feasible strategy.

Chun's team also is looking for other genes that HIF2A may target in $\mathrm{OA}$, whether other HIF proteins regulate HIF2A expression or play a role in OA and whether HIF2A contributes to rheumatoid arthritis (RA).

He said the Gwangju Institute of Science and Technology has filed a patent application for the findings.

Chun added that the team is interested in licensing or partnering arrangements, especially those that would aid the discovery and development of HIF2A inhibitors.

Haas, M.J. SciBX 3(22); doi:10.1038/scibx.2010.663

Published online June 3, 2010

\section{REFERENCES}

1. Saito, T. et al. Nat. Med.; published online May 23, 2010; doi:10.1038/nm.2146

Contact: Katsushi Tokunaga, The University of Tokyo, Tokyo, Japan e-mail: kawaguchi-ort@h.u-tokyo.ac.jp

2. Yang, S. et al. Nat. Med.; published online May 23, 2010; doi:10.1038/nm.2153

Contact: Jang-Soo Chun, Gwangju Institute of Science and Technology, Gwangju, South Korea e-mail: jschun@gist.ac.kr

COMPANIES AND INSTITUTIONS MENTIONED

Ajou University, Suwon, South Korea

Gwangju Institute of Science and Technology, Gwangju, South Korea University Hospital Muenster, Muenster, Germany

The University of Tokyo, Tokyo, Japan

Wonkwang University Medical Center, Iksan, South Korea 